\title{
Adult Obstructive Cor Triatriatum with Severe Mitral Regurgitation:
}

\section{A Case Report}

\author{
Tomomi Nakajima, MD, ${ }^{1}$ Dung Van Hung, MD, PhD, ${ }^{1,2}$ Yuji Hiramatsu, $\mathrm{MD}, \mathrm{PhD}^{3}$ \\ 'Department of Cardiac Surgery, Heart Institute of Ho Chi Minh City-Alan Carpentier Foundation, Ho Chi Minh City, Vietnam; \\ ${ }^{2}$ Department of Thoracic and Cardiovascular Surgery, University of Medicine Pham Ngoc Thach, Ho Chi Minh City, Vietnam; \\ ${ }^{3}$ Department of Cardiovascular Surgery, Faculty of Medicine, University of Tsukuba, Tsukuba, Japan
}

\section{ABSTRACT}

Adult cor triatriatum sinister associated with severe mitral regurgitation is extremely rare. As these obstructive cor triatriatum feature hemodynamics that mimic mitral stenosis, a pressure load is theoretically generated only on the left atrial proximal chamber, and therefore the left ventricle is less likely to suffer volume loading. Here, we report a surgical case with such rare hemodynamics. A 22-year-old man with obstructive cor triatriatum and severe mitral regurgitation received an anomalous membrane excision and mitral annuloplasty. An abnormal membrane with an orifice $7 \mathrm{~mm}$ in size was completely resected while a grossly dilated mitral annulus was repaired via annuloplasty ring. Mitral regurgitation was controlled well, and the postoperative course was uneventful. Even with obstructive cor triatriatum, severe mitral annular dilatation and subsequent left ventricular dilatation may occur, causing the progressive heart failure encountered in this case.

\section{INTRODUCTION}

Cor triatriatum sinister (CTS) is a rare congenital heart anomaly consisting of an abnormal fold of tissue, a membrane, or a fibromuscular band that subdivides the left atrium (LA) into two chambers [Niwayama 1960; Rodefeld 1990]. CTS usually presents in childhood, and the size of the membrane orifice determines the degree of hemodynamic severity, which typically mimics mitral stenosis (MS). In this report, we present a very rare adult case of obstructive CTS concomitant with severe mitral regurgitation (MR) as well as annular and left ventricular $(\mathrm{LV})$ dilatation. The patient underwent successful definitive repair with mitral annuloplasty.

\section{CASE REPORT}

A 22-year-old man experiencing progressive fatigue was referred to our department for CTS and severe MR. Although

Received April 6, 2021; accepted May 3, 2021.

Correspondence: Tomomi Nakajima, MD, Department of Cardiac Surgery, Heart Institute of Ho Chi Minh City-Alan Carpentier Foundation, 4 Duong Quang Trung, District 10, Ho Chi Minb City, Vietnam; +84-38-651-586; fax: +84-38-651-543 (e-mail: tnakajima-tuk@umin.ac.jp).

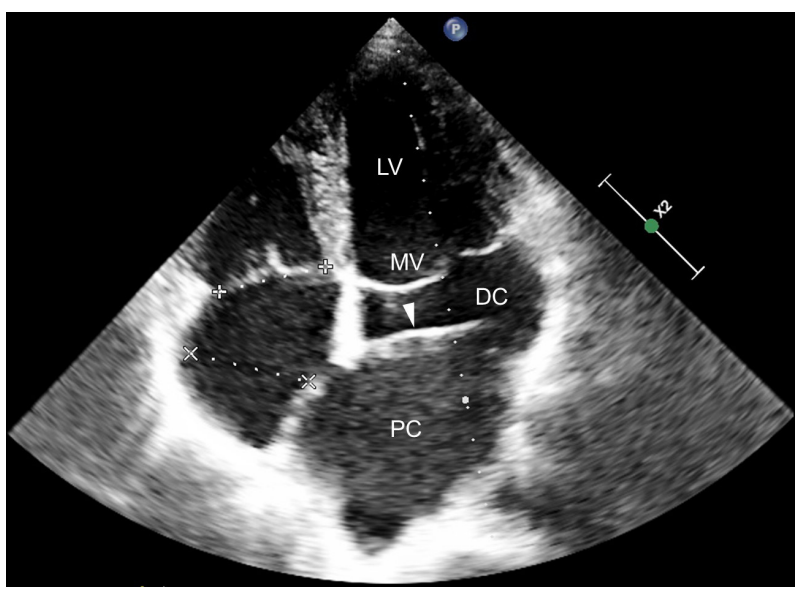

Figure 1. Preoperative echocardiography showing an anomalous membrane (white arrow) in the left atrium that divided it into distal and proximal chambers. The left atrium was dilated to $52 \mathrm{~mm}$. Although the mitral annulus was grossly dilated to $55 \mathrm{~mm}$, no obvious mitral valve prolapse was seen. The left ventricle was dilated to $61 \mathrm{~mm}$ in diastole and $42 \mathrm{~mm}$ in systole. Ejection fraction was $58 \%$. DC, distal chamber; LV, left ventricle; MV, mitral valve; PC: proximal chamber

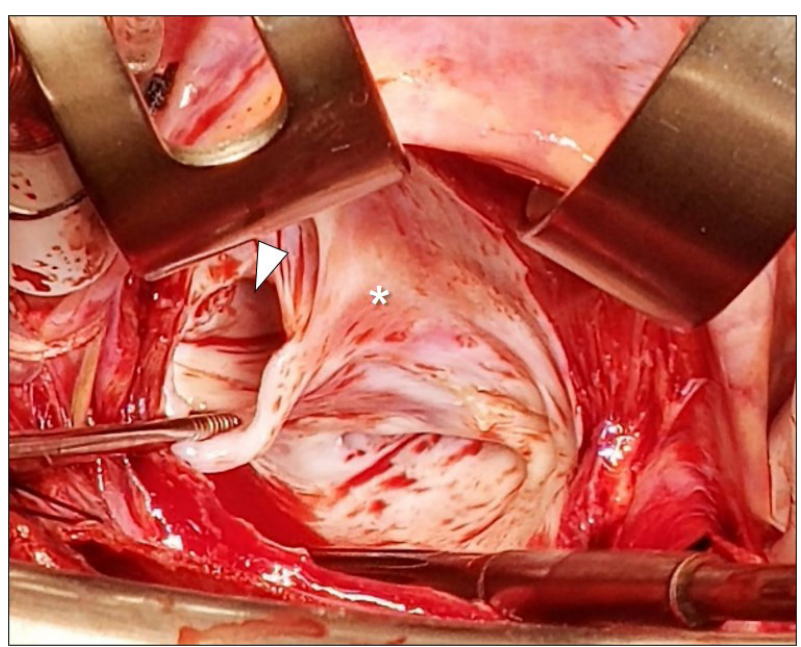

Figure 2. Intra-operative finding. The anomalous membrane $\left(^{*}\right)$, revealed after opening the proximal chamber. The orifice (white arrow) on the membrane resembled an oval flap and was hidden behind folds. The mitral valve was observable only after the membrane was resected. 
his medical history included mild exercise intolerance from early childhood and paroxysmal atrial fibrillation (PAF) diagnosed three years prior, he previously had never been admitted or treated. Echocardiography revealed an anomalous membrane in the LA that created a two-chambered separation, suggestive of obstructive CTS, with a $7 \mathrm{~mm}$ slit-like orifice (Figure 1) and a concomitant dilated LV (LV diastolic diameter $61 \mathrm{~mm}, \mathrm{LV}$ systolic diameter $42 \mathrm{~mm}$ ) and LA (LA diameter $52 \mathrm{~mm}$ ). The ejection fraction was $58 \%$ and the mitral annulus was extremely dilated to $55 \mathrm{~mm}$ with severe regurgitation, while systolic pulmonary arterial pressure was measured at $45 \mathrm{mmHg}$.

Intraoperative findings revealed that all pulmonary veins were connected to a proximal chamber of the LA in which the fossa ovalis was located. The orifice of the abnormal membrane was hidden behind a flap-like fold and resembled a slit with a long axis of $7 \mathrm{~mm}$ on first sight but became oval shaped when the flap was tensioned up. (Figure 2) The absence of a congenital left-to-right shunt led to the diagnosis of Lucas \& Schmidt classification type IA. The anomalous membrane then was completely resected. Although the mitral valve had a severely dilated annulus, no prolapse or dysplasia of the leaflets was observed and a simple mitral ring annuloplasty was performed with a $36 \mathrm{~mm}$ Carpentier-Edwards Physio II Annuloplasty Ring (Edwards Lifesciences, Irvine, CA). No anti-arrhythmic procedures were conducted for PAF, but the postoperative course was uneventful, and discharge occurred on postoperative day 6. Echocardiography upon discharge showed well-controlled MR and no pulmonary hypertension.

\section{DISCUSSION}

Since obstructive CTS usually is fatal before adulthood, encounters in adults are unusual. The mechanism for symptom onset in adults is the development of supra-mitral inflow obstruction, due to progressive fibrosis and calcification of the anomalous orifice, mitral regurgitation, pulmonary hypertension, atrial fibrillation, or combinations of these [Rudiene 2019; Eichholz 2013].

CTS associated with severe MR is rarely reported, with only 13 previous case reports (most of which are in adults), and reports in juveniles are even scarcer [Rodefeld 1990]. Within this limited case pool, obstructive CTS with severe MR due to a grossly dilated mitral annulus, as seen in the present case, is extremely rare [Jayaprakash 2015; Wong 1989; Nakajima 2002]. Of note in our case, the mitral leaflets had nearly normal configuration and only the annular dilatation was suspected as causative for the MR. It is unclear why the annular dilatation with enlarged $\mathrm{LV}$ progressed since obstructive CTS is less likely to cause LV volume loading and only the proximal chamber receives pressure loading. We suppose that obstructive CTS caused dilatation of the highpressure proximal chamber, resulting in whole LA dilatation followed by gross mitral annular dilatation. Although the precise pathogenesis and onset of the mitral annular dilatation is unclear due to a lack of childhood medical records, the present case details suggest that, under the MS-like conditions seen in CTS, mitral annular dilatation and LV overload can readily develop. In addition, once MR occurs, the regurgitative flow may interfere with the forward flow through the narrow membrane orifice, worsening the hemodynamics and compounding the MS-like effect [Fujii 2020]. This chronic mechanism may be causative for the adult-onset, progressive heart failure seen in our case.

In conclusion, the coexistence of obstructive CTS and severe mitral regurgitation, due to severe annular dilatation, is a significant discovery that warrants vigilance. Cardiac surgeons also should be aware that this rare adult hemodynamic status, featuring CTS behaving as both MS and MR, can be encountered.

\section{REFERENCES}

Eichholz JL, Hodroge SS, Crook II JJ, Mack Jr JW, Wortham DC. 2013. Cor triatriatum sinister in a 43-year-old man with syncope. Tex Heart Inst J 40:602-5.

Fujii S, Gottschalk BH, Iglesias I, Evans B, Hage A, Chu MWA. 2020. Endoscopic repair of obstructive cor triatriatum. CASE (Phila) 4:82-5.

Jayaprakash K, Madhavan S, Kumary VS, George R, Cletus N, Baiju S. 2015. Wong's anomaly - A rare variant of cor triatriatum. Indian Heart J 67:469-71.

Nakajima H, Kobayashi J, Kurita T, Kitamura S. 2002. Maze procedure and cor triatriatum repair. Ann Thorac Surg 74:251-3.

Niwayama G. 1960. Cor triatriatum (review). Am Heart J 59:291-317.

Rodefeld MD, Brown JW, Heimansohn DA, et al. 1990. Cor triatriatum: clinical presentation and surgical results in 12 patients. Ann Thorac Surg 50:562-8.

Rudiene V, Hjostshoj CMS, Glaveckaite S, et al. 2019. Cor triatriatum sinistrum diagnosed in the adulthood: a systematic review. Heart. 105:1197-202.

Wong CK, Leung WH, Cheng CH, Lau CP, Cheung DLC. 1989. Myxomatous mitral valve degeneration complicating asymptomatic cor triatriatum. Clin Cardiol. 12:48-50. 